
\section{BMJ Quality}

\title{
Reducing CBC Clotting Rates in the Neonatal Patient Care Areas
}

\author{
Jennifer McCoy, Tanya Tichon, Michael Narvey
}

To cite: McCoy J, Tichon T, Narvey M. Reducing CBC Clotting Rates in the Neonatal Patient Care Areas. BMJ Quality Improvement Reports 2016;5:u215456.w4946. doi:10.1136/bmjquality. u215456.w4946

Received 25 May 2016 Revised 29 June 2016

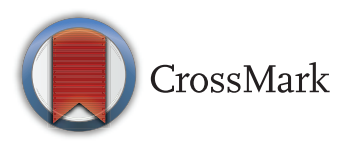

Health Sciences Centre, Canada

Correspondence to Jennifer McCoy jmccoy@exchange.hsc.mb.ca

\section{ABSTRACT}

Performing a complete blood count (CBC) is a common test performed in neonatal intensive care. Samples reported as "clotted" are not able to be analyzed and require redraw. A perceived "high" clotting rate elicits frustration among team members and has negative effects on patient flow and patient satisfaction. Process mapping and a root cause analysis determined that an educational intervention was required to optimize blood collection skills of front-line nurses. Through four rapid PDSA cycles over a three year period, the neonatal patient care areas were able to decrease their CBC clotting rates from $30 \%$ (monthly rate when the problem was identified) to $16 \%$ (yearly average at the end of the project). The $\mathrm{CBC}$ clotting rates continue to decease over time due to the integration of a multi-faceted educational plan into biannual education days designed for current staff nurses, as well as into the orientation plan for newly hired and student nurses.

\section{PROBLEM}

The Neonatal Patient Care areas at the Health Sciences Centre in Winnipeg, Manitoba, Canada are divided into a Level 2 and Level 3 Nursery: Intermediate Care Nursery (IMCN), and the Neonatal Intensive Care Unit (NICU), respectively. The IMCN is a 24 bed unit located in close proximity to the Women's Hospital where approximately 5700 deliveries occur each year. The NICU has 17 beds, and is located in the Children's Hospital. Between both units, approximately 1,100 infants receive care on an annual basis.

On July 1st, 2012, the Laboratory Service Technicians discontinued providing services, including capillary blood sampling, or "heel pokes" to the Intermediate Care Nursery. The rationale for this change was driven by the importance of cue-based care and protecting the premature or sick neonates' sleep by avoiding set times for blood draws. Cue-based care is a principle employed by many NICUs where infants are handled during their quiet-alert or active states. This is a relatively new method of providing care to the sick or preterm infant designed to protect their growing central nervous system.
Historically, infants were woken routinely at 0700 to have blood drawn by the lab, regardless of where the infant was in their sleep cycle. Thereafter, bedside nurses were required to perform capillary blood sampling when appropriate as determined by the principles of developmental care. However, as a psychomotor skill, capillary blood sampling was not easily transferred to a new set of hands. A common blood test performed in the NICU is a complete blood count (CBC). Once the blood has been collected it is sent to the lab for analysis. One reason the lab may not be able to perform the analysis is because the platelets in the blood have caused the sample to clot. This results in the nurse having to re-draw the sample from the infant. A unit's "clotting rate" is the percentage of clotted samples out of the total number of samples drawn in any given month. The bedside nurses in both units became frustrated, as they perceived their CBC clotting rates as "high" in comparison to the state before the change. Others queried whether clotting rates had always been "high" and were now being highlighted since each nurse now followed up their own sample result. These concerns and unanswered questions led the nurse educator to delve into a system-wide analysis, extensive educational roll-out, and rapid PDSA (plan-do-study-act) cycle execution.

\section{BACKGROUND}

Discharges, transfers to the healthy maternalnewborn care areas, and treatment decisions are all impacted by the results of blood work and tests. Blood work is deliberately sampled and collected in the early hours of the morning in order to facilitate results being available for interpretation by the team during interdisciplinary rounds held at 0900 . When clotting occurs, the sample must be re-collected leading to delays in the decisions described above, and interrupts patient flow. In addition, the frequent skin breaks inhibits the care team's ability to provide appropriate cue-based care. 
Another concern stems from the potential to impact long term outcomes for the neonate. While beside nurses use a plethora of non-pharmacological methods to reduce the pain experienced by a capillary blood sample (for example, sucrose, containment, positioning, skin-to-skin, breast-feeding), the procedure breaks the skin and elicits pain. A second sampling required is just as painful as the first. Pain in the preterm infant can have long lasting consequences such as neurodevelopmental impairment, altered response to pain as adults and internalizing behaviours. ${ }^{1}$ Non pharmacologic measures to reduce pain such as skin-to-skin care reduce the incidence of apnea as well as many significant morbidities in the NICU. ${ }^{2}$ Conversely, untreated pain in the preterm or sick neonate may increases oxygen requirements, apnea and bradycardia and ultimately lead to a longer duration of treatments such as ventilation (invasive or non-invasive), supplemental oxygen, or caffeine administration. ${ }^{3}$ In addition to potentially delaying discharge, these treatments are not without risks themselves, as for example increasing risks of retinopathy of prematurity from increased oxygen administration. ${ }^{4}$ While a second capillary blood sampling may often be perceived as benign, it's important to consider the cumulative effects of multiple recollections.

\section{BASELINE MEASUREMENT}

In May 2012, prior to the discontinuation of laboratory services, the clotting rate in IMCN was $16 \%$ while that in NICU during the same time period was $17 \%$. In July of the same year after the bedside nurses were assigned the responsibility of capillary blood sampling the CBC clotting rate increased to $32 \%$ for the Intermediate Care Nursery and $30 \%$ for the Neonatal Intensive Care Unit. This $100 \%$ increase in IMCN and $76 \%$ increase for NICU in CBC sample clotting rates validated the bedside nurses' concerns and provided the units with a baseline and motivation to improve upon these results. At the same time, the laboratory began to use new sampling tubes to facilitate mass sample processing by machine rather than by hand. This was the result of their own cost-saving process improvement project. However, little education was provided to front line staff regarding this change.

\section{DESIGN}

The team leader of this project was the nurse educator. Other team members included the patient care managers from both units, a clinical practice consultant from the laboratory, a laboratory information management specialist who provided the team with the monthly clotting rates, and a product representative for the company that supplies the blood collection tubes and the lancets used to break the skin. After mapping out the process of blood collection, sample delivery, analysis, and results reporting in the neonatal patient care areas the nursing educator focused her efforts on assessing sample collection and implementing interventions to improve successful initial blood draws from neonatal patients by the bedside nurses. Over the next three years, psychomotor education of bedside nurses with extensive collaboration of several stakeholders was the primary intervention utilized to achieve the desired goal of decreasing clotting rates in the neonatal patient care areas.

In terms of a SMART aim, our goal was to have the neonatal bedside nurses responsible for collecting blood samples for CBC testing, decrease their clotting rates from $30 \%$ observed in August 2012 to $7-10 \%$, as was the case in a another neonatal intensive care unit in our city.

\section{STRATEGY}

\section{Cycle 1}

Plan: The first intervention was the development of a joint plan between nursing and laboratory staff to assess and address potential contributing factors. The discussion between the laboratory personnel and nursing revealed that a well-mixed CBC sample would not clot, regardless of the length of time between collection and analysis. This finding reinforced the appropriateness of focusing intervention on collection methods and the skills of the bedside nurse, rather than investigating transport processes such as Patient Transport and the pneumatic tube system.

Do: The joint plan was communicated via an action plan newsletter developed by the nurse educator and disseminated by email and print copy to all patient care areas. As a second step, consultation with a product specialist from the manufacturer of the capillary blood collection tubes offered additional advice on best practice which was then communicated to nursing at educational sessions consisting of "hands-on" practice, information posters, and a representative from the manufacturer to answer questions posed by staff. This model of education delivery facilitated effective practice change in a variety of learning domains.

Study: Less than one month after these sessions were complete, representatives from the manufacturer came to the IMCN and NICU to directly observe the bedside nurses' collection methods and provided real-time, face-to-face feedback to the staff. Regular data collection of CBC clotting rates began.

Act: The observations of laboratory staff informed the team that further education, hands on training, and monitoring of $\mathrm{CBC}$ clotting rates was needed to affect positive change.

Cycle 2

Plan: Following the production of the November 2012 action plan from the first PDSA cycle, a product change was introduced. The Quickheel Lancet $\bigcirc$ was announced as the primary device for accessing a patient's capillary blood supply. To maintain staff engagement, updated monthly reports were circulated to nursing, indicating the clotting rates for both units which further contained summaries of practice changes from the PDSA cycles. 


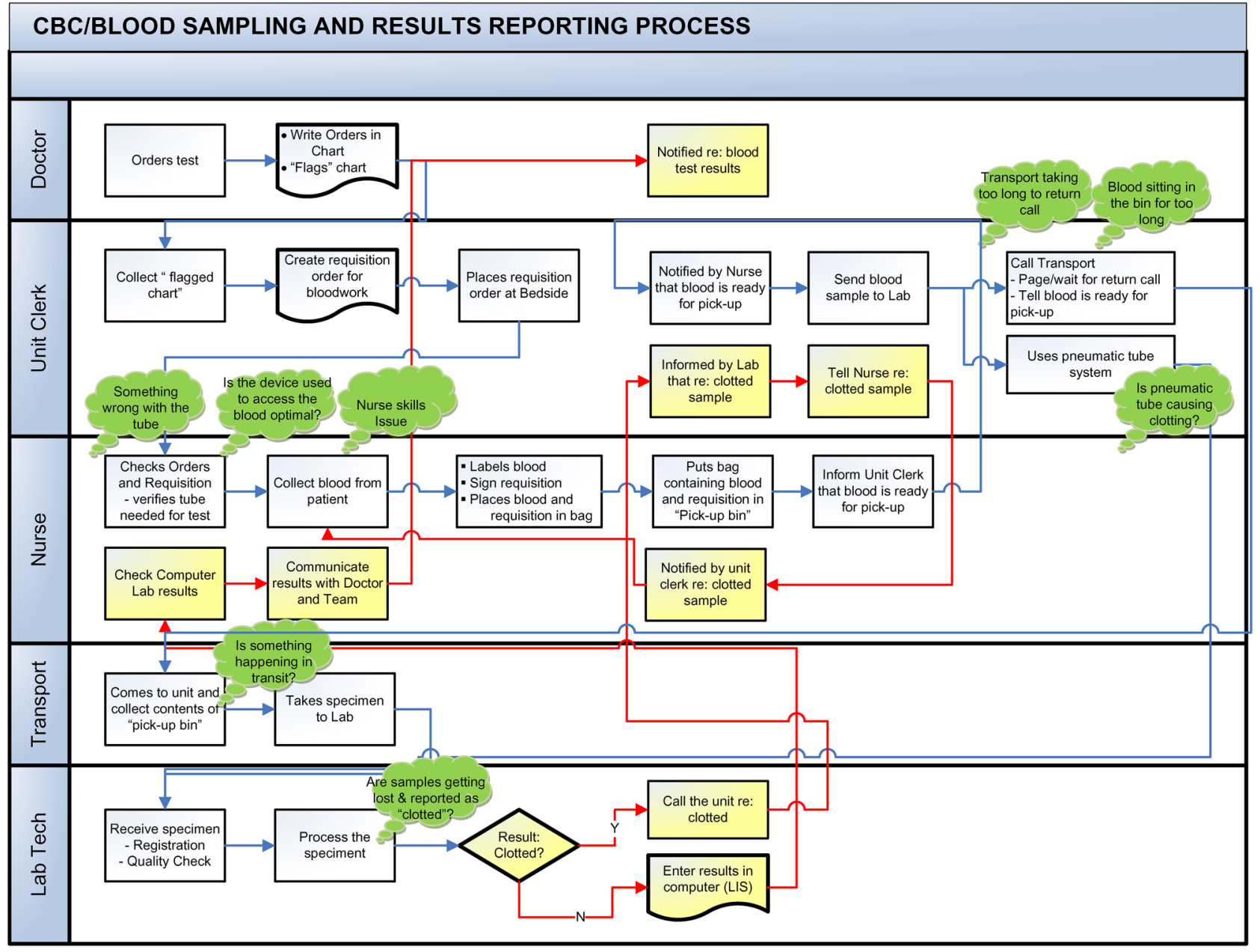

Figure 1 Blood Collection Process Map

Demonstrating positive change with their efforts was deemed essential to facilitate acceptance of the Quickheel Lancet@. In January 2013 a meeting between staff and the hospital Quality and Safety team culminated in a decision to obtain more frequent data.

Do: Following the meeting with hospital Quality and Safety team, the laboratory agreed to provide weekly clotting rates to the neonatal patient care units. The nursing leadership team began to encourage bedside and charge nurses to complete Patient Safety Event Reports each time a sample was received as clotted thereby cognitively escalating this issue as a true safety issue in the eyes of staff. Another update was circulated to staff.

Study: The nurse educator networked with the other level III NICU in the city (St. Boniface Hospital) in May 2013 to obtain a comparison data set. The neighboring unit was found to have significantly lower CBC clotting rates (2\%-8\% compared with Health Sciences Centre's $16 \%-27 \%$ for the same time period). It was found that St. Boniface Hospital still utilized Laboratory Technicians for capillary blood sampling. The technician team was small, potentially allowing greater opportunity for each technician to practice their technique and become proficient.
Act: The weekly clotting rate reports and the comparison with the other hospital informed the team that further gains in decreasing clotting rates may yet be attainable as the bedside staff become skilled in capillary blood sampling.

Cycle 3

Plan: From June 2013 to the start of the next PDSA cycle in June 2014, combined clotting rates had returned to near baseline at $20 \%$. One third of the time, the combined clotting rates were less than $20 \%$. In June of 2014, the nurse educator visited St. Boniface Neonatal Intensive Care Unit again and directly observed capillary blood sampling techniques performed by their experienced laboratory technicians. The nurse educator on this occasion recognized additional practice differences, the most important of which was pre-warming of the infant's limb prior to performing the skin break.

Do: The practice differences observed at St. Boniface were communicated in conjunction with a practice change recommendation to all staff at Health Sciences Centre neonatal patient care areas. In October 2014, a representative from the supplier of the collection tubes returned to provide guidance and a "hands on" 


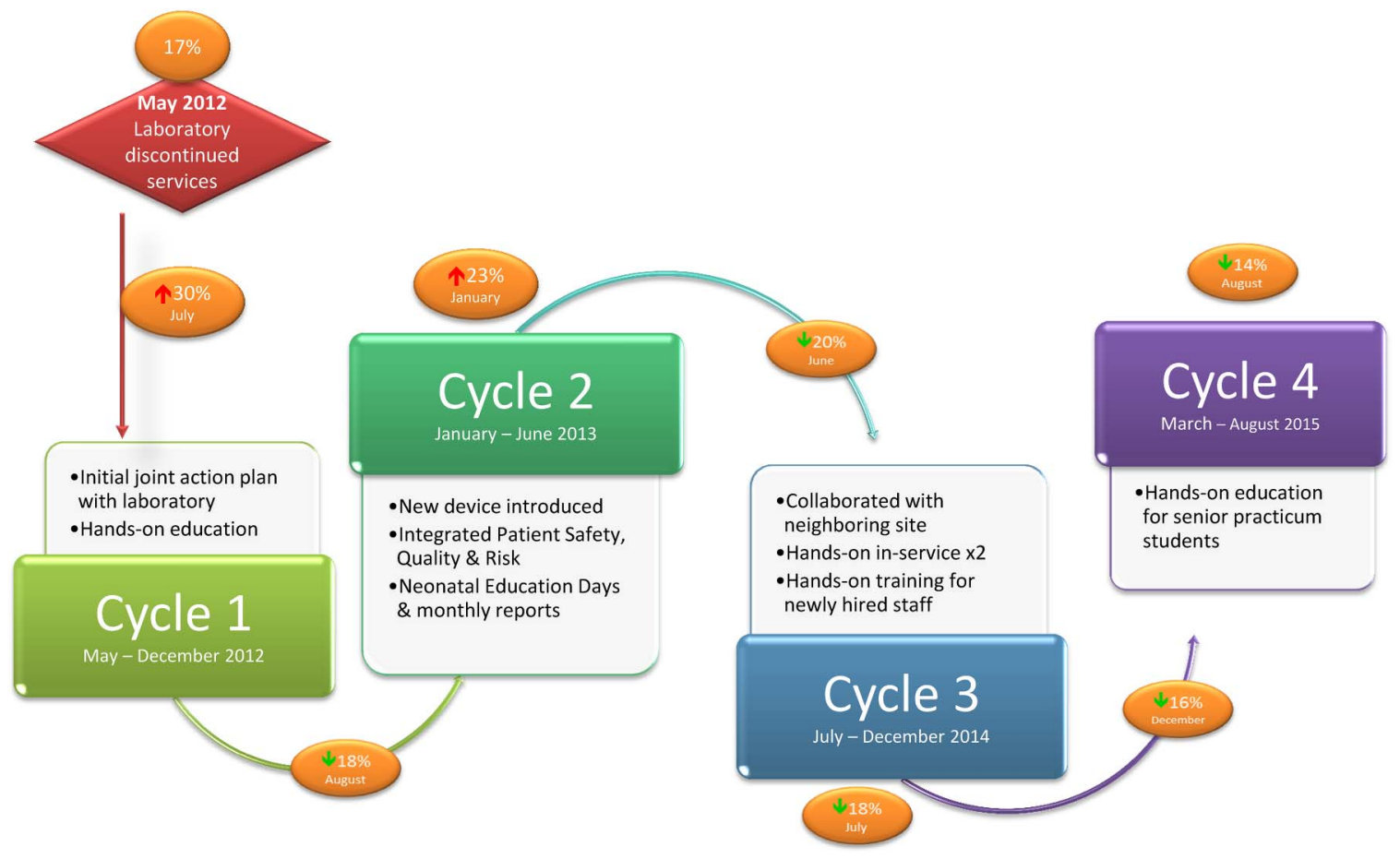

Figure 2 PDSA Map

demonstration to many of the new hires since the start of the improvement project.

Study: Ongoing clotting rate reports were being provided to the team and circulated to the bedside staff to facilitate engagement in the project.

Act: Improvements in clotting rates continued to be seen. The median clotting rate over the span of the third PDSA cycle was $18 \%$. This was the first time since the beginning of the project where the median for the cycle was less than $20 \%$.

Cycle 4

Plan: In the March of 2015, it was identified that nursing students completing their senior practicum in the neonatal patient care areas were not provided with the same educational package during their training. Many of these students are hired into the NICU and IMCN environment and therefore a possible gap might exist prior to them receiving scheduled education to reduce clotting.

Do: Training for senior practicum students was therefore added to ensure that from the start, any care provider who may practice capillary blood sampling was equipped the necessary skill set.

Study: The Laboratory Information Management Specialist continued to provide the nurse educator with monthly clotting rates for both units. The nurse educator in turn disseminates this information to front line staff.

Act: Hands-on education for senior practicum staff and newly hired staff has been sustained since this project.

\section{RESULTS}

Post Measurement

Cycle 1

The evaluation of the first PDSA cycle occurred in November 2012 with all pertinent findings and recommendations emailed to all of the bedside nurses. Appendix 2 contains a copy of the communication staff received. The action plan highlighted CBC clotting rates for both IMCN and NICU. During the planning phase in August and September the units experienced an increase from $18 \%$ to $22 \%$ overall clotting rates combined. After the data collection and correspondence to nursing was completed the rate returned to a combined rate of $19 \%$ for both units. This recovery was interpreted as a success and validated the practice recommendations and the benefit of hands-on in-services.

Cycle 2

In June 2013 at the end of this PDSA cycle, clotting rates were reported to the front line staff showing a downward trend and bolstering support for the initiative. For the entire year of 2013, combined rates for the NICU and IMCN were as low as $16 \%$ (mean 22\%) and a lowest month's rate in the NICU of $9 \%$. Reinforcement of this initiative continued, with monthly clotting reports and twice yearly educational sessions for nursing that remain in place.

Cycle 3

As a result of the efforts of this cycle, the combined CBC clotting rates for November and December 2014 were $17 \%$ and $16 \%$ respectfully. Between June and December, 
inclusive, the combined $\mathrm{CBC}$ clotting rates were less than $20 \%$ more than half of the time.

\section{Cycle 4}

Combined unit CBC clotting rates fell from a baseline of $21 \%, 18 \%$, and $21 \%$ for January, February, and March, 2015 respectively to $14 \%, 13 \%$, and $7 \%$ for October, November, and December, 2015. The 2015 yearly average was $16 \%$. The lowest monthly clotting rate was comparable to those of St. Boniface Hospital NICU at $6 \%$. This rate was achieved by both neonatal patient care areas independently (IMCN in December, 2015 and by the NICU in September, 2015).

This project had little to no costs associated with its implementation. Existing resources were utilized and expanded upon to optimize their value. Resources used included the nurse educator, Neonatal Education Days, hands-on in-services, and newsletter communication. The human resources such as the nurse educator, specialists from the manufacturer of the collection tubes, laboratory technicians, and others who were consulted incorporated this project into their existing workload. No additional persons were hired for the project. The purchase of the Quickheel Lancet( $\bigcirc$ replaced another product. Therefore, the net cost was virtually zero and was part of an existing cost centre budget.

In addition to the project having little if any expense, the consequential decrease in clotted CBC samples reduced overall spending in the neonatal patient care areas. Fewer repeated sample collection decreases the average number of supplies used per patient and positively effects the bedside nurse productivity since less time is spent repeating tasks. In terms of patient flow, a reduction in clotted CBC samples, increases the number of samples' results available for interpretation at a convenient and appropriate time for care providers. The readiness of results expedites decision making related to discharge, transfer, or further investigations.

See supplementary file: ds8731.doc - "BMJ Appendices"

\section{LESSONS AND LIMITATIONS}

Prior to using a formalized approach with sequential PDSA cycles, staff assumed that high CBC clotting rates were related to Laboratory or Patient Transport processes. After completing the process map and investigating those areas of concern, we discovered that these perceptions could not be supported. As the extensive educational plan was implemented, it became apparent that improving the blood sampling skills of the bedside nurses yielded the greatest positive change. This rigorous process highlighted to all staff the importance of Quality Improvement in the region.

The importance of ongoing diverse education cannot be underappreciated. The demographics of the approximately 200 bedside nurses in the neonatal patient care areas varied widely. Both novice and expert nurses benefited from the expansive approaches taken to address this issue. The education provided to nurses addressed the three domains of learning: cognitive, affective, and psychomotor. ${ }^{5}$ Auditory, visual, and kinesthetic learners were supported through the varied media and tools used to present information such as email, newsletter, posters, diagrams, and physical props. Learning in different styles was also facilitated by the different forums to address the target audience such as large, small, and one-on-one groups as well as indirect communication. In terms of generalizability, while the CBC clotting rate issue was unit-specific, the methods used to address the problem could be applied to many psychomotor skills in the health care field, regardless of specialty and patient population. The breadth of educational strategies used would likely yield comparable success for other teams.

Maintaining these results will take continued determination by the health care team. From May 2012 to March 2016, the NICU collected an average of 124 samples for CBC analysis per month, while the IMCN, 84. During these years the monthly sample totals showed a downward trend as strategies to minimize blood sampling in our smallest infants took hold. In an era of declining opportunities to practice proper technique it will be imperative to ensure continuance of the developed educational strategies to prevent creep towards rates seen prior to the intervention. As shown in figure 1 the median demonstrated a gradual reduction in clotted samples with each PDSA cycle. In spite of declining sample collections, analysis of the last PDSA cycle time period suggests an accelerated decrease in clotting rates compared to the slow and steady linear decrease experienced in the first three PDSA cycles. As shown in figure 1 in the first three PDSA cycles there was significant variation in clotting rates as evidenced by the low $\mathrm{R}$ values of $0.1632,0.0866$, and 0.1354 respectively. More consistent reductions were noted after the last PDSA cycle (March 2015 to April 2016 inclusive) with a line of best fit having a higher R2 of 0.6331. It is unclear whether the improvement in consistency was due to a greater contribution of the strategies employed in cycle 4 or the cumulative efforts of all four cycles but this reduction in variability has persisted since the conclusion of the last PDSA cycle.

As this project only sought to address clotting from capillary samples we do not have data demonstrating comparative rates for venipuncture and central line access. Although questioned by our health care team, data collection to address this began, but was never reviewed. If concerns arise again in the future regarding the clotting rates of CBC samples, this may be an avenue to explore.

\section{CONCLUSION}

As part of the obtaining background data, we discovered that CBC clotting rates for the site as a whole are greater when compared to neighboring acute care centres in the city. Other departments such as Children's 
Emergency Department and the ambulatory care areas have also completed process maps for blood collection and results reporting. These departments are primarily focusing on the transit time between the nurse or phlebotomist obtaining the sample from the patient and the appropriate care provider receiving the results. In addition to CBC clotting, these areas express frustration with high hemolysis of electrolyte samples. The work the neonatal patient care areas did to improve their clotting rates and subsequently, patient flow and client experience may serve as a foundation for other departments to strive for the same improvements. While the CBC samples collected from neonates are unique due to the use of capillary blood sampling, the educational framework used to affect change and monitoring strategies are not. Other departments and patient care areas, regardless of population demographic, can benefit from the lessons learned in the neonatal patient care areas at Health Sciences Centre.

After collaboration with all stake holders, an action plan was formed and re-formed several times over to address an issue identified by front-line staff caring for the most vulnerable patients. Hands-on small-group or one-on-one teaching was done in the clinical area three times. The issue, recommendations, and progress to date has been presented to staff at their Neonatal Education Days seven times between 2012 and 2015 and continues to be included in the sessions held twice yearly. Progress is reported to staff on a monthly basis. The target audience has expanded beyond employed registered nurses to include student nurses completing their senior practicum in the neonatal patient care areas. The combined effort of these initiatives has produced a significant and sustainable change in the clotting rates of $\mathrm{CBC}$ samples drawn by bedside nurses in the neonatal population at the Health Sciences Centre. Due to the positive outcomes yielded by this project, evidence now exists to frame future quality improvement initiatives where staff education is pivotal to success.

Acknowledgements

- Shelley Probizanski, Manager, Quality, Patient Safety \& Risk

- Sybil Russell RN BN, Patient Care Manager

- Alun Carter, Laboratory Information Management Specialist

- Jenny Dimanauhan, Quality Analysist

- Susan Csatari RN, BD Diagnostic \& Prenalytical Systems

- Jennifer Prichodko, BD Medical Surgical Systems

Declaration of interests The authors have no conflicts of interest to declare.

Ethical approval Ethical review was not sought since the project was one of quality improvement and was therefore not required by the institution. Patients were not subjected to more or less testing than was deemed medically necessary as per the Health Sciences Centre usual ethically approved practices.

Open Access This is an open-access article distributed under the terms of the Creative Commons Attribution Non-commercial License, which permits use, distribution, and reproduction in any medium, provided the original work is properly cited, the use is non commercial and is otherwise in compliance with the license. See:

- $\mathrm{http}: / /$ creativecommons.org/licenses/by-nc/2.0/

- http://creativecommons.org/licenses/by-nc/2.0/legalcode

\section{REFERENCES}

1. Ranger M, Synnes AR, Vinall J, Grunau RE. Internalising behaviours in school-aged children born very premature are predicted by neonatal pain and morphine exposure. European Journal of Pain 2014;18:844-52.

2. Conde-Aqudelo A, Diaz-Rossello JL. Kangaroo mother care to reduce morbidity and mortality in low birthweight infants. Cochrane Database Systematic Review 2014;16.

3. Brummelte S, Grunau RE, Chau V, Poskitt KJ, Brant R, Vinall J, Gover A, Synnes AR, Miller SP. Procedural pain and brain development in premature newborns. Ann Neurol. 2012;71: 385-96.

4. Carlo WA, Bell E, Walsh M. Target ranges of oxygen saturation in extremely preterm infants. N Engl J Med 2010;362:1959-69.

5. Bloom BS, Engelhart MD Furst EJ, Hill WH, Krathwohl DR. Taxonomy of educational objectives: The classification of educational goals. Handbook l: Cognitive domain. New York: David McKay Company; 1956. 